\title{
STABILITY OF TAUTOLOGICAL BUNDLES ON THE HILBERT SCHEME OF TWO POINTS ON A SURFACE
}

\author{
MALTE WANDEL
}

\begin{abstract}
Let $(X, H)$ be a polarized smooth projective surface satisfying $H^{1}\left(X, \mathcal{O}_{X}\right)=0$, and let $\mathcal{F}$ be either a rank 1 torsion-free sheaf or a rank 2 $\mu_{H}$-stable vector bundle on $X$. Assume that $c_{1}(\mathcal{F}) \neq 0$. This article shows that the rank 2-respectively, rank 4 - tautological sheaf $\mathcal{F}^{[2]}$ associated with $\mathcal{F}$ on the Hilbert square $X^{[2]}$ is $\mu$-stable with respect to a certain polarization.
\end{abstract}

\section{$\S 0$. Introduction}

Let $X$ be an algebraic K3 surface with polarization $H \in \operatorname{Pic} X$, and let $v=(r, c, s) \in \mathbb{N} \oplus \mathrm{NS}(X) \oplus \mathbb{Z}$. Mukai has shown $[\mathrm{M}]$ that in many cases - if $v$ is carefully chosen - the moduli space of $H$-semistable sheaves of rank $r$, first Chern class $c$, and second Chern class $s$, is again a smooth compact complex manifold carrying a holomorphic symplectic structure. In fact, all these moduli spaces are deformation-equivalent to $\operatorname{Hilb}^{n}(X)$ for some $n \geq 0$. Now the natural question arises: What happens if we start with another hyperkähler manifold (instead of a K3 surface) and study the geometry of moduli spaces of sheaves on this manifold? Not much is known about this topic, and one of the fundamental questions is the following: Does there exist a symplectic structure on these moduli spaces? Of course, answering this question in general will be very complicated. But one could hope to at least find an example of such a moduli space that does carry such a symplectic structure. Therefore, we need examples of vector bundles on higher-dimensional hyperkähler manifolds, and then we have to inquire about the stability of these bundles. One big class of examples are the so-called tautological bundles on the Hilbert schemes of K3 surfaces. They arise as the images of vector bundles on a K3 under a Fourier-Mukai transform. We will concentrate on the case of $\operatorname{Hilb}^{2}(X)$, where $X$ is a projective K3-surface. Schlickewei

Received May 23, 2012. Revised May 22, 2013. Accepted May 30, 2013.

First published online February 24, 2014.

2010 Mathematics Subject Classification. Primary 14J60; Secondary 14D20, 14J28, $14 \mathrm{~F} 05$. 
[Sch] has shown that in many cases tautological bundles associated with line bundles on $X$ are stable with respect to a carefully chosen polarization on the Hilbert scheme. We will extend this result by showing that, in fact, every rank 2 tautological sheaf associated with any rank 1 torsion-free sheaf having nonvanishing first Chern class is stable with respect to some polarization. Furthermore, we will prove that the rank 4 tautological vector bundle associated with any stable rank 2 bundle is stable. Again, we assume that the first Chern class is nontrivial. This provides us with quite a large variety of stable vector bundles on $\operatorname{Hilb}^{2}(X)$. A forthcoming paper [W] will show that in some cases the component of the moduli space of sheaves on $\mathrm{Hilb}^{2}(X)$ containing the tautological sheaves is smooth and isomorphic to the moduli space of sheaves on the K3 surface. It is therefore an irreducible holomorphic symplectic manifold.

In fact, all results concerning the stability of the tautological sheaves are valid for any smooth projective surface $X$ satisfying $h^{1}\left(X, \mathcal{O}_{X}\right)=0$, so they will be presented in this generality.

\section{Notation and convention}

- In this article, all schemes and varieties will be defined over the field of complex numbers.

- For a vector bundle $\mathcal{E}$, we write $\mathbb{P}(\mathcal{E})$ for $\operatorname{Proj}\left(\operatorname{Sym}\left(\mathcal{E}^{\vee}\right)\right)$ following the definition in Fulton's textbook [F, Appendix B5.5]. In this definition, $\mathbb{P}(\mathcal{E})$ is the bundle of lines of $\mathcal{E}$.

- By $A^{\star}(Y)$ we denote the Chow ring of any smooth projective variety $Y$.

\section{$\S 1$. The geometric setup}

Let $X$ be a projective surface satisfying $h^{1}\left(X, \mathcal{O}_{X}\right)=0$, and choose a polarization $H$. Throughout this text we will consider the following basic blowup and projections diagram:

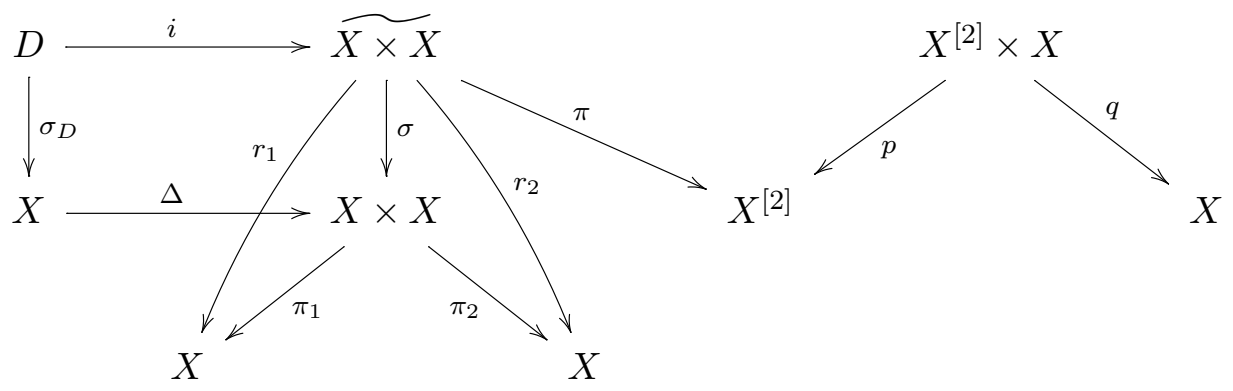


Here $\Delta$ is the diagonal embedding, $\sigma$ is the blowing-up morphism (we are blowing up the diagonal $)$, and $D \simeq \mathbb{P}\left(\mathcal{N}_{X \mid X \times X}\right) \simeq \mathbb{P}\left(\mathcal{T}_{X}\right)$ denotes the exceptional divisor together with the projection $\sigma_{D}$, the inclusion $i$, and $\mathcal{O}_{D}(1)$, the dual of the tautological line bundle. It is well known that $\mathcal{N}_{D \mid \widetilde{X \times X}} \cong \mathcal{O}_{D}(-1)$ (see, e.g., [H, Chapter II, Theorem 8.24c]). Furthermore, $\pi_{1}, \pi_{2}, p$, and $q$ denote the natural projections onto the particular factors, and $r_{1}$ and $r_{2}$ are the compositions of $\pi_{1}$ and $\pi_{2}$ with $\sigma$. Last but not least, we have the flat 2-to-1 covering $\pi$.

We will continue with some considerations concerning the Picard groups of the varieties we are looking at. Note that by the assumption that $h^{1}(X$, $\left.\mathcal{O}_{X}\right)=0$, a line bundle is uniquely determined by its first Chern class. For the same reason, this holds true for $X \times X$ and $\widetilde{X \times X}$. We will therefore often use the same notation for a line bundle as for the corresponding classes in the Chow ring and cohomology.

We have $\operatorname{Pic}(X \times X) \cong(\operatorname{Pic} X)^{\boxplus 2}$. Here we apply [H, Exercise III, 12.6b] since $h^{1}\left(X, \mathcal{O}_{X}\right)=0$. And accordingly, we have $\operatorname{Pic}(\widetilde{X \times X}) \cong(\operatorname{Pic} X)^{\boxplus 2} \oplus$ $\mathbb{Z} D$. We will write an element of $\operatorname{Pic}(\widetilde{X \times X})$ as $g \otimes 1+1 \otimes h+a D$ for some $g, h \in \operatorname{Pic} X$ and $a \in \mathbb{Z}$, and we denote the corresponding line bundle by $\mathcal{L}_{(g, h, a)}$.

Furthermore, it is well known that $\operatorname{Pic} X^{[2]} \cong \operatorname{Pic} X \oplus \mathbb{Z} \delta$, where $\delta$ is a class such that $2 \delta$ is the exceptional divisor in $X^{[2]}$ coming from the blowup of the diagonal in the quotient $(X \times X) / S_{2}$. We will denote the line bundle corresponding to $\delta$ by $\mathcal{L}_{\delta}$. Thus, we can write every element in $\operatorname{Pic}\left(X^{[2]}\right)$ as $\mathcal{L}_{X} \otimes \mathcal{L}_{\delta}^{\otimes a}$ for some $\mathcal{L}_{X} \in \operatorname{Pic} X$ and $a \in \mathbb{Z}$. Note that with this notation we have $\pi^{\star} \mathcal{L}_{X}=\mathcal{L}_{(l, l, 0)}$ for a line bundle $\mathcal{L}_{X}$ on $X$ with first Chern class $l$. Furthermore, we have the relations $\pi^{\star} \delta=D$ and $\pi_{\star} D=2 \delta$.

Next let us summarize the most important facts about the Chow rings of the varieties involved in the upper diagram. We will follow very closely [F, Sections 6.7 and 15.4], especially [F, Lemma 15.4]. On $D=\mathbb{P}\left(\mathcal{T}_{X}\right)$ we have the short exact sequence: $0 \rightarrow \mathcal{O}_{D}(-1) \rightarrow \sigma_{D}^{\star} \mathcal{N}_{X \mid X \times X} \rightarrow \mathcal{Q} \rightarrow 0$, where $\mathcal{Q}$ is the universal quotient line bundle. We have $\mathcal{N}_{X \mid X \times X} \simeq \mathcal{T}_{X}$, and by comparing Chern classes, we can see that $\mathcal{Q} \simeq \mathcal{O}_{D}(1) \otimes \sigma_{D}^{\star} \omega_{X}^{\vee}$ :

$$
0 \rightarrow \mathcal{O}_{D}(-1) \rightarrow \sigma_{D}^{\star} \mathcal{T}_{X} \rightarrow \mathcal{O}_{D}(1) \otimes \sigma_{D}^{\star} \omega_{X}^{\vee} \rightarrow 0
$$

Let $\xi$ denote the first Chern class of $\mathcal{O}_{D}(1)$. By [F, Remark 3.2.4 and Theorem 3.3] we have

$$
A^{*}(D) \cong A^{*}(X)[\xi] /\left(\xi^{2}+c_{1}\left(\mathcal{T}_{X}\right) \xi+c_{2}\left(\mathcal{T}_{X}\right)\right)
$$


Furthermore, [F, Proposition 6.7e] describes the structure of $A^{*}(\widetilde{X \times X})$. We gather the most important identities in this ring in the following lemma.

Lemma 1.1. Let $\alpha, \beta, \gamma \in A^{*}(X)$. In $A^{*}(\widetilde{X \times X})$ we have the following identities:

(a)

$$
i_{\star}\left(\xi \cdot \sigma_{D}^{\star}(\alpha)\right)=\sigma^{\star} \Delta_{\star}(\alpha)+i_{\star} \sigma_{D}^{\star}\left(\alpha \cdot \omega_{X}\right),
$$

(b)

$$
i^{\star} i_{\star} \lambda=-\xi \cdot \lambda \quad \text { for all } \lambda \in A^{*}(D),
$$

(c)

$$
i_{\star} \sigma_{D}^{\star} \alpha \cdot \sigma^{\star}(\beta \otimes \gamma)=i_{\star} \sigma_{D}^{\star}(\alpha \cdot \beta \cdot \gamma),
$$

(d)

$$
i_{\star} \sigma_{D}^{\star}(\alpha) \cdot i_{\star} \sigma_{D}^{\star}(\beta)=-\sigma^{\star} \Delta_{\star}(\alpha \cdot \beta)-i_{\star} \sigma_{D}^{\star}\left(\alpha \cdot \beta \cdot \omega_{X}\right) .
$$

Proof.

(a) This follows from the general formula in [F, Proposition 6.7.a]. Note that in this case the excess normal bundle is just the universal quotient bundle denoted by $\mathcal{Q}$ above. We have $c_{1}(\mathcal{Q})=c_{1}\left(\mathcal{O}_{D}(1) \otimes \sigma_{D}^{\star} \omega_{X}^{\vee}\right)=$ $\xi-\sigma_{D}^{\star} \omega_{X}$.

(b) This is the self-intersection formula in [F, Corollary 6.3]:

$$
i^{\star} i_{\star} \lambda=c_{1}\left(\mathcal{N}_{D \mid \widetilde{X \times X}}\right) \cdot \lambda=c_{1}\left(\mathcal{O}_{D}(-1)\right) \cdot \lambda=-\xi \cdot \lambda .
$$

(c) We have $\alpha \cdot \beta \cdot \gamma=\alpha \cdot \Delta^{\star}(\beta \otimes \gamma)$. Applying $\sigma_{D}^{\star}$, we get

$$
\begin{aligned}
\sigma_{D}^{\star}(\alpha \cdot \beta \cdot \gamma) & =\sigma_{D}^{\star}\left(\alpha \cdot \Delta^{\star}(\beta \otimes \gamma)\right) \\
& =\sigma_{D}^{\star} \alpha \cdot \sigma_{D}^{\star} \Delta^{\star}(\beta \otimes \gamma)=\sigma_{D}^{\star} \alpha \cdot i^{\star} \sigma^{\star}(\beta \otimes \gamma)
\end{aligned}
$$

Now we apply $i_{\star}$ and use the projection formula.

(d) We use the projection formula and then (b) to find

$$
\begin{aligned}
i_{\star} \sigma_{D}^{\star}(\alpha) \cdot i_{\star} \sigma_{D}^{\star}(\beta) & =i_{\star}\left(i^{\star} i_{\star} \sigma_{D}^{\star}(\alpha) \cdot \sigma_{D}^{\star}(\beta)\right) \\
& =-i_{\star}\left(\xi \cdot \sigma_{D}^{\star}(\alpha) \cdot \sigma_{D}^{\star}(\beta)\right)=-i_{\star}\left(\xi \cdot \sigma_{D}^{\star}(\alpha \cdot \beta)\right) .
\end{aligned}
$$

Now we apply (a) and we are done. 
Corollary 1.2. We have

(a)

$$
i^{\star} D=-\xi
$$

where we denote $i_{\star}[D] \in A^{3}(\widetilde{X \times X})$ simply by $D$;

(b)

$$
D^{2}=-i_{\star} \xi=-\sigma^{\star} \Delta-i_{\star} \sigma_{D}^{\star}\left(\omega_{X}\right)
$$

where $\Delta$ also denotes the cohomology class of the diagonal in $X \times X$;

(c)

$$
\left(\sigma^{\star} \Delta\right)^{2}=\sigma^{\star} \Delta_{\star}\left(c_{2}\left(\mathcal{T}_{X}\right)\right) .
$$

Proof. (a) Apply (b) of the lemma to $\lambda=[D]$.

(b) We use (a), and for the second equality we apply (a) of the lemma to $\alpha=[X]$ to get

$$
D^{2}=i_{\star} i^{\star} D=i_{\star}(-\xi)=-\sigma^{\star} \Delta-i_{\star} \sigma_{D}^{\star}\left(\omega_{X}\right)
$$

(c) In a very similar manner to the proof of (b) in the lemma, we use the self-intersection formula:

$$
\begin{aligned}
\left(\sigma^{\star} \Delta\right)^{2} & =\sigma^{\star}\left(\Delta^{2}\right)=\sigma^{\star} \Delta_{\star} \Delta^{\star} \Delta=\sigma^{\star} \Delta_{\star} \Delta^{\star} \Delta_{\star}[X] \\
& =\sigma^{\star} \Delta_{\star}\left(c_{2}\left(\mathcal{N}_{X \mid X \times X}\right)\right)=\sigma^{\star} \Delta_{\star}\left(c_{2}\left(\mathcal{T}_{X}\right)\right) .
\end{aligned}
$$

Let us finish this section by determining the canonical line bundles of $D$ and $\widehat{X \times X}$. On $\widetilde{X \times X}$, we have a short exact sequence

$$
0 \rightarrow \mathcal{T}_{\widetilde{X \times X}} \rightarrow \sigma^{\star} \mathcal{T}_{X \times X} \rightarrow i_{\star}\left(\mathcal{O}_{D}(1) \otimes \sigma_{D}^{\star} \omega_{X}^{\vee}\right) \rightarrow 0
$$

We immediately see that $c_{1}\left(\mathcal{T}_{\widetilde{X \times X}}\right)=r_{1}^{\star} c_{1}\left(\mathcal{T}_{X}\right)+r_{2}^{\star} c_{1}\left(\mathcal{T}_{X}\right)-D$, and therefore, that $\omega_{\widetilde{X \times X}}=\mathcal{L}_{\left(\omega_{X}, \omega_{X}, 1\right)}$.

Next, on $D$ we have the exact sequence

$$
0 \rightarrow \mathcal{T}_{D} \rightarrow i^{\star} \mathcal{T}_{\widetilde{X \times X}} \rightarrow \mathcal{O}_{D}(-1) \rightarrow 0
$$

Again, we derive $c_{1}\left(\mathcal{T}_{D}\right)=2 \sigma_{D}^{\star} c_{1}\left(\mathcal{T}_{X}\right)+2 \xi$, so $\omega_{D} \simeq \sigma_{D}^{\star}\left(\omega_{X}^{\vee}\right)^{\otimes 2} \otimes \mathcal{O}_{D}(-2)$. 


\section{$\S 2$. Tautological bundles}

Now let $\mathcal{F}$ be a vector bundle on $X$ of rank $r$ with first Chern class $f$. Recall that in $X^{[2]} \times X$ there is the universal subscheme $\Xi$ consisting of pairs $(\xi, x)$ such that $x \in \xi$. We define the tautological bundle associated with $\mathcal{F}$ to be the image of $\mathcal{F}$ under the Fourier-Mukai transform with the structure sheaf of the universal subscheme as kernel:

$$
\mathcal{F}^{[2]}:=R p_{\star}\left(q^{\star} \mathcal{F} \otimes \mathcal{O}_{\Xi}\right)
$$

Since we are considering only the case of the second Hilbert scheme, we can simplify this definition. Indeed, the universal subscheme $\Xi$ is isomorphic to the blowup $\widetilde{X \times X}$ of $X \times X$ along the diagonal. (A detailed discussion of this fact can be found in [EGL, Section 1]). Via this isomorphism, $p$ restricted to $\Xi$ corresponds to the 2 -to- 1 cover $\pi$, and $q$ corresponds to the morphism $r_{1}=\sigma \circ \pi_{1}$. Thus, we end up with the much simpler formula

$$
\mathcal{F}^{[2]}=\pi_{\star} r_{1}^{\star} \mathcal{F}
$$

REMARK. We see immediately that this process is, in fact, an exact functor, and we do not need to derive the pushforward along the finite morphism $\pi$.

Now $\mathcal{F}^{[2]}$ is, of course, a vector bundle on $X^{[2]}$ of rank $2 r$, and we have the following formula for its dual.

Lemma 2.1. Let $\mathcal{F}$ be a vector bundle on $X$. Then

$$
\left(\mathcal{F}^{[2]}\right)^{\vee} \simeq \mathcal{F}^{\vee[2]} \otimes \mathcal{L}_{\delta}
$$

Proof. Using the Grothendieck-Verdier duality, we have

$$
\begin{aligned}
\mathcal{F}^{[2]} \vee & =\mathcal{H o m}_{\mathcal{O}_{X[2]}}\left(\pi_{\star} r_{1}^{\star} \mathcal{F}, \mathcal{O}_{X[2]}\right) \simeq \pi_{\star} \mathcal{H} \operatorname{lom}_{\mathcal{O}_{\overline{X \times X}}}\left(r_{1}^{\star} \mathcal{F}, \mathcal{L}_{\left(\omega_{X}, \omega_{X}, 1\right)} \otimes \pi^{\star} \omega_{X}^{\vee[2]}\right) \\
& \simeq \pi_{\star} \mathcal{H} \operatorname{Hom}_{\widetilde{\mathcal{O}_{X \times X}}}\left(r_{1}^{\star} \mathcal{F}, \mathcal{L}_{(0,0,1)}\right) \simeq \pi_{\star}\left(r_{1}^{\star} \mathcal{F}^{\vee} \otimes \pi^{\star} \mathcal{L}_{\delta}\right) \\
& \simeq \mathcal{F}^{\vee[2]} \otimes \mathcal{L}_{\delta} .
\end{aligned}
$$

Note that we use here the fact that in the identification $\operatorname{Pic}\left(X^{[2]}\right) \cong \operatorname{Pic}(X) \oplus$ $\mathbb{Z} \delta$, we have $\omega_{X^{[2]}} \simeq \omega_{X}$. 
The pullback $\pi^{\star} \mathcal{F}^{[2]}$ of a tautological sheaf fits into a basic exact sequence as follows:

$$
0 \rightarrow \pi^{\star} \mathcal{F}^{[2]} \rightarrow r_{1}^{\star} \mathcal{F} \oplus r_{2}^{\star} \mathcal{F} \rightarrow i_{\star} \sigma_{D}^{\star} \mathcal{F} \rightarrow 0
$$

This sequence was already used by Danila [D] and Schlickewei [Sch] to study tautological sheaves, and they provide a basic proof of its existence. Furthermore, note that the exactness of this sequence is a special case of a more general result due to Scala (see $[\mathrm{Sc}]$ ). We want to deduce a simple formula for the first Chern class of $\pi^{\star} \mathcal{F}^{[2]}$. We start with a definition.

Definition 2.2. Let $Y$ be a smooth projective variety, and let $\mathcal{E}$ be a sheaf on $Y$ with $\operatorname{dim} \operatorname{Supp} \mathcal{E} \leq d$. Let $Z_{i}$ be the irreducible components of the support of $\mathcal{E}$ of dimension $d$, and denote by $r_{i}$ the generic rank of $\mathcal{E}$ on $Z_{i}$. We define the $d$-cycle associated with $\mathcal{E}$ to be $Z_{d}(\mathcal{E}):=\sum_{i} r_{i}\left[Z_{i}\right]$.

Proposition 2.3. Let $Y$ be a smooth projective variety, let $i$ : $W \hookrightarrow Y$ be a closed subscheme of dimension $d$, and let $\mathcal{E}$ be a sheaf on $W$. We have

$$
\operatorname{ch}\left(i_{\star} \mathcal{E}\right)=Z_{d}\left(i_{\star} \mathcal{E}\right)+\text { terms of higher codimension (TOHC). }
$$

Proof. This follows from the generalized Grothendieck-Riemann-Roch theorem as stated in [F, Section 18.3]. By [F, Example 18.3.11], we have

$$
\tau_{Y}\left(i_{\star} \mathcal{E}\right)=Z_{d}\left(i_{\star} \mathcal{E}\right)+\mathrm{TOHC} .
$$

Now we use [F, Theorem 18.3] to proceed:

$$
\begin{aligned}
\tau_{Y}\left(i_{\star} \mathcal{E}\right) & =\operatorname{ch}\left(i_{\star} \mathcal{E}\right) \cap \tau_{Y}\left(\mathcal{O}_{Y}\right)=\left(\operatorname{ch}_{d}\left(i_{\star} \mathcal{E}\right)+\mathrm{TOHC}\right) \cap([Y]+\mathrm{TOHC}) \\
& =\operatorname{ch}_{d}\left(i_{\star} \mathcal{E}\right)+\mathrm{TOHC} .
\end{aligned}
$$

For the first inequality, we use [F, Theorem 18.3(2)], and for the second, [F, Theorem 18.3(5)]. Note that since $Y$ is smooth, $i_{\star} \mathcal{E}$ admits a locally free resolution.

Corollary 2.4. We have

$$
c_{1}\left(\pi^{\star} \mathcal{F}^{[2]}\right)=r_{1}^{\star} f+r_{2}^{\star} f-r D .
$$

In the following we will analyze conditions such that $\mathcal{F}^{[2]}$ is stable. In order for this to be possible, we will from now on assume that we are given 
a polarization $H$ of $X$ and that $\mathcal{F}$ is $\mu_{H}$-stable. More precisely, for every subsheaf $\mathcal{E} \subseteq \mathcal{F}$ of rank $0<r_{\mathcal{E}}<r$, we have

$$
\frac{c_{1}(\mathcal{E}) \cdot H}{r_{\mathcal{E}}}<\frac{c_{1}(\mathcal{F}) \cdot H}{r}
$$

Next, we have to fix a polarization on $X^{[2]}$. This is done as follows. For $N \in \mathbb{N}$ we define $H_{N}:=N H-\delta$. (Recall that we always use the identification $\operatorname{Pic}\left(X^{[2]}\right) \cong \operatorname{Pic} X \oplus \mathbb{Z} \delta$.) This divisor is ample for all sufficiently large $N$, say, $N \geq N_{0}$.

Now let us assume that there is a destabilizing subsheaf $\mathcal{E}^{\prime} \subseteq \mathcal{F}^{[2]}$. Pulling back both sheaves via $\pi$, we get an inclusion of sheaves on $\widehat{X \times X}$ :

$$
\pi^{\star} \mathcal{E}^{\prime}=: \mathcal{E} \subseteq \pi^{\star} \mathcal{F}^{[2]} .
$$

Since the slope of a vector bundle is just multiplied by 2 under the finite pullback $\pi^{\star}, \mathcal{E}$ is also a destabilizing subsheaf of $\pi^{\star} \mathcal{F}^{[2]}$ with respect to the polarization $\widetilde{H_{N}}=\pi^{\star} H_{N}$ of $\widetilde{X \times X}$. Therefore, we will, in fact, consider destabilizing subbundles of $\pi^{\star} \mathcal{F}^{[2]}$ which come from $X^{[2]}$.

As a first step toward any considerations about the stability of a vector bundle $\pi^{\star} \mathcal{F}^{[2]}$, we first have to calculate the slope of a sheaf $\mathcal{E}$ with respect to the given polarization. It is defined as

$$
\mu_{\widetilde{H_{N}}}(\mathcal{E}):=\frac{c_{1}(\mathcal{E}){\widetilde{H_{N}}}^{3}}{r_{\mathcal{E}}},
$$

considered as a number by integrating against the fundamental class of $\widetilde{X \times X}$. Thus, we first calculate the expansion of ${\widetilde{H_{N}}}^{3}$ :

$$
\begin{aligned}
{\widetilde{H_{N}}}^{3}= & (N H \otimes 1+1 \otimes N H-D)^{3} \\
= & (N H \otimes 1+1 \otimes N H)^{3}-3(N H \otimes 1+1 \otimes N H)^{2} D+O(N) \\
= & 3 N^{3}\left(H^{2} \otimes H+H \otimes H^{2}\right)-3 N^{2}\left(H^{2} \otimes 1+2 H \otimes H+1 \otimes H^{2}\right) D \\
& +O(N) .
\end{aligned}
$$

Now let $\mathcal{E}$ be a sheaf on $\widetilde{X \times X}$. We write its first Chern class as $c_{1}(\mathcal{E})=$ $g \otimes 1+1 \otimes h+a D$, with $g, h \in \operatorname{Pic} X$ and $a \in \mathbb{Z}$. 
Lemma 2.5. Let $\mathcal{F}$ be a sheaf on $X$ of rank $r$, and let $\mathcal{E}$ be a sheaf on $\widetilde{X \times X}$ of rank $r_{\mathcal{E}}$. We have the following expansions for the slopes of $\mathcal{E}$ and $\pi^{\star} \mathcal{F}^{[2]}$ :

$$
\begin{aligned}
\mu_{\overparen{H_{N}}}(\mathcal{E}) & =\frac{1}{r_{\mathcal{E}}}\left\{3 H^{2}(H .(g+h)) N^{3}+12 a H^{2} N^{2}\right\}+O(N), \\
\mu_{\widetilde{H_{N}}}\left(\pi^{\star} \mathcal{F}^{[2]}\right) & =\frac{3 H^{2}(H . f)}{r} N^{3}-6 H^{2} N^{2}+O(N) .
\end{aligned}
$$

Proof. First, note that (5) is just the special case of setting $g=h=$ $f, a=-r$, and $r_{\mathcal{E}}=2 r$ in (4). Next, from Lemma 1.1(c) we deduce that $\sigma^{\star}\left(A^{i}(X \times X)\right) \cdot i_{\star} \sigma_{D}^{\star}\left(A^{j}(X)\right)=0$ for $i+j>4$. Thus, half of the terms in our computation vanish, and we are left with

$$
\begin{aligned}
{\widetilde{H_{N}}}^{3} c_{1}(\mathcal{E})= & 3 N^{3}\left(H^{2} \otimes H+H \otimes H^{2}\right)(g \otimes 1+1 \otimes h) \\
& -3 N^{2}\left(H^{2} \otimes 1+2 H \otimes H+1 \otimes H^{2}\right) D \cdot a D+O(N) .
\end{aligned}
$$

Finally, note that by Lemma 1.1 and Corollary 1.2, we have

$$
\begin{aligned}
\left(H^{2} \otimes 1\right) D^{2} & =-\left(H^{2} \otimes 1\right) \sigma^{\star} \Delta-\left(H^{2} \otimes 1\right) i_{\star} \sigma_{D}^{\star} \omega_{X} \\
& =-H^{2}-i_{\star} \sigma_{D}^{\star}(\underbrace{H^{2} \cdot \omega_{X}}_{=0}) \\
& =-H^{2},
\end{aligned}
$$

and similarly for the terms with $H \otimes H$ and $1 \otimes H$.

\section{$\S 3$. Destabilizing line subbundles of tautological bundles}

In this section we will show that, for $N \geq N_{0}$, there exist no $H_{N}$-destabilizing line subbundles $\mathcal{L}^{\prime} \subseteq \mathcal{F}^{[2]}$ in the case $\mathcal{F} \not \mathcal{O}_{X}$. We assume that $\mathcal{L}^{\prime}$ is such a destabilizing line subbundle. The pullback $\mathcal{L}=\pi^{\star} \mathcal{L}^{\prime}$ of such a line bundle is a destabilizing line subbundle of the pullback $\pi^{\star} \mathcal{F}^{[2]}$ with respect to $\widetilde{H_{N}}$. Composing this inclusion with the one from the basic exact sequence (3), we find that $\mathcal{L} \subseteq r_{1}^{\star} \mathcal{F} \oplus r_{2}^{\star} \mathcal{F}$. We will proceed by showing that $\operatorname{Hom}_{\widetilde{X \times X}}\left(\mathcal{L}, r_{i}^{\star} \mathcal{F}\right)=0$ for $i=1,2$. The situation is completely symmetric; thus we will focus on $\operatorname{Hom}_{\widetilde{X \times X}}\left(\mathcal{L}, r_{1}^{\star} \mathcal{F}\right)$. We write the first Chern class of $\mathcal{L}$ as $c_{1}(\mathcal{L})=g \otimes 1+1 \otimes h+a D$, with $g=c_{1}(\mathcal{G})$ and $h=c_{1}(\mathcal{H})$ for some line bundles $\mathcal{G}$ and $\mathcal{H}$ on $X$. In fact, since $\mathcal{L}$ is coming from $X^{[2]}$, this class is invariant under the $S_{2}$-action; that is, $g=h$. But for later use we will 
proceed in this generality and denote the line bundle class with first Chern class equal to $g \otimes 1+1 \otimes h+a D$ simply by $\mathcal{L}_{(g, h, a)}$. We have the following central result.

Proposition 3.1. For all $g, h \in \operatorname{Pic} X$ and for all $a \in \mathbb{Z}$, we have

$$
\operatorname{Hom}_{\widetilde{X \times X}}\left(\mathcal{L}_{(g, h, a)}, r_{1}^{\star} \mathcal{F}\right) \subseteq \operatorname{Hom}_{X}(\mathcal{G}, \mathcal{F})^{h^{2}\left(X, \mathcal{H} \otimes \omega_{X}\right)} .
$$

Proof. Consider the defining exact sequence of the structure sheaf of the exceptional divisor $D$ :

$$
0 \rightarrow \mathcal{L}_{(0,0,-1)} \rightarrow \mathcal{O}_{\widetilde{X \times X}} \rightarrow \mathcal{O}_{D} \rightarrow 0
$$

Tensoring this sequence with $\mathcal{L}_{(0,0, a)}$, we have

$$
0 \rightarrow \mathcal{L}_{(0,0, a-1)} \rightarrow \mathcal{L}_{(0,0, a)} \rightarrow \mathcal{O}_{D}(-a) \rightarrow 0
$$

so we see immediately that $\sigma_{\star} \mathcal{L}_{(0,0, a)}$ is contained in $\mathcal{O}_{X \times X}$ for all $a \in \mathbb{Z}$. Thus, we find that $r_{1 \star}\left(r_{2}^{\star} \mathcal{H}^{\vee} \otimes \mathcal{L}_{(0,0,-a)}\right) \simeq \pi_{1 \star}\left(\pi_{2}^{\star} \mathcal{H}^{\vee} \otimes \sigma_{\star} \mathcal{L}_{(0,0,-a)}\right)$ is a subsheaf of $\pi_{1 \star} \pi_{2}^{\star} \mathcal{H}^{\vee} \simeq H^{0}\left(\mathcal{H}^{\vee}\right) \otimes \mathcal{O}_{X} \simeq \mathcal{O}_{X}^{h^{2}\left(X, \mathcal{H} \otimes \omega_{X}\right)}$. Now using the projection formula and adjunction, we get

$$
\begin{aligned}
\operatorname{Hom}_{\widetilde{X \times X}}\left(\mathcal{L}_{(g, h, a)}, r_{1}^{\star} \mathcal{F}\right) & \cong \operatorname{Hom}_{\widetilde{X \times X}}\left(r_{1}^{\star} \mathcal{G}, r_{1}^{\star} \mathcal{F} \otimes \mathcal{L}_{(0,-h,-a)}\right) \\
& \cong \operatorname{Hom}_{X}\left(\mathcal{G}, r_{1 \star}\left(r_{1}^{\star} \mathcal{F} \otimes \mathcal{L}_{(0,-h,-a)}\right)\right) \\
& \cong \operatorname{Hom}_{X}\left(\mathcal{G}, \mathcal{F} \otimes r_{1 \star}\left(r_{2}^{\star} \mathcal{H}^{\vee} \otimes \mathcal{L}_{(0,0,-a)}\right)\right)
\end{aligned}
$$

Together with the inclusion above, we are done.

Corollary 3.2. Let $\mathcal{F}$ be a $\mu_{H}$-stable vector bundle on $X$ of rank $r$ and first Chern class $c_{1}(\mathcal{F})=f$. Then $r_{1}^{\star} \mathcal{F}$ contains no line subbundles $\mathcal{L}_{(g, h, a)}$ satisfying

$$
H .(g+h) \geq \frac{H . f}{r},
$$

except the case $r=1, h=0$, and $g=f$.

Proof. Let $\mathcal{L}_{(g, h, a)}$ be a line subbundle of $r_{1}^{\star} \mathcal{F}$ satisfying the hypothesis of the corollary. We will show that $\operatorname{Hom}_{X}(\mathcal{G}, \mathcal{F})^{h^{2}\left(X, \mathcal{H} \otimes \omega_{X}\right)}=0$, which yields a contradiction to Proposition 3.1.

If $H . h>0$, we have $0=h^{0}\left(X, \mathcal{H}^{\vee}\right)=h^{2}\left(X, \mathcal{H} \otimes \omega_{X}\right)$, and we are done. 
If $H . h \leq 0$, we see that

$$
H . g \geq H .(g+h) \geq \frac{H . f}{r},
$$

so if $\mathcal{G} \not \mathcal{F}$ by the stability of $\mathcal{F}$, we have $\operatorname{Hom}_{X}(\mathcal{G}, \mathcal{F})=0$.

If $\mathcal{G} \simeq \mathcal{F}$, we must have $r=1$ and equalities everywhere in (9), so $H . h=0$. But then again, $h^{2}\left(X, \mathcal{H} \otimes \omega_{X}\right)=0$ for all such $\mathcal{H}$ but the trivial line bundle, that is, $h=0$.

Now that we have an explicit description for possible homomorphisms from a line bundle to $\pi^{\star} \mathcal{F}^{[2]}$, let us take a closer look at the destabilizing condition for line subbundles in $\pi^{\star} \mathcal{F}^{[2]}$.

Lemma 3.3. For sufficiently large $N$, a line subbundle $\mathcal{L}_{(g, h, a)}$ in $\pi^{\star} \mathcal{F}^{[2]}$ is $H_{N}$-destabilizing if

$$
r H .(g+h)>H . f \quad \text { or } \quad r H .(g+h)=H . f \quad \text { and } \quad a \geq 0 .
$$

Proof. Equation (4) in Lemma 2.5 computes the expansion of the slope of $\mathcal{L}_{(g, h, a)}$ as

$$
\mu_{\widetilde{H_{N}}}\left(\mathcal{L}_{(g, h, a)}\right)=3 H^{2}(H \cdot(g+h)) N^{3}+12 a H^{2} N^{2}+O(N) .
$$

And we also derived the expansion of $\mu_{\widetilde{H_{N}}}\left(\pi^{\star} \mathcal{F}^{[2]}\right)$ in equation (5) in Lemma 2.5:

$$
\mu_{\widetilde{H_{N}}}\left(\pi^{\star} \mathcal{F}^{[2]}\right)=\frac{3 H^{2}(H . f)}{r} N^{3}-6 H^{2} N^{2}+O(N) .
$$

Thus, $\mathcal{L}_{(g, h, a)}$ is destabilizing if either $r H .(g+h)>H . f$ or $r H .(g+h)=H . f$ and $2 a>-1$. Since $a \in \mathbb{Z}$, we can replace the last inequality by $a \geq 0$.

TheOREM 3.4. Let $\mathcal{F}$ be a $\mu_{H}$-stable vector bundle on $X$ of rank $r$ and first Chern class $c_{1}(\mathcal{F})=f$. Assume that $\mathcal{F} \not \mathcal{O}_{X}$. Then for sufficiently large $N$, the tautological vector bundle $\mathcal{F}^{[2]}$ on $X^{[2]}$ has no $\mu_{H_{N}}$-destabilizing line subbundles.

Proof. As explained before, we are reduced to considering an $S_{2}$-equivariant destabilizing line subbundle $\mathcal{L}_{(g, g, a)}$ of $\pi^{\star} \mathcal{F}^{[2]}$. The destabilizing condition yields $H . g \geq H . f / 2 r$, so by Corollary 3.2 , such a line subbundle cannot exist. 


\section{$\S 4$. The cases $r=1$ and $r=2$}

From Theorem 3.4 we deduce the following.

Corollary 4.1. Let $\mathcal{F}$ be a line bundle on $X$ not isomorphic to $\mathcal{O}_{X}$. Then for sufficiently large $N$, bundle $\mathcal{F}^{[2]}$ is a $\mu_{H_{N}}$-stable rank 2 vector bundle on $X^{[2]}$.

Proof. Since $\mathcal{F}^{[2]}$ has rank 2 , we have only to consider torsion-free destabilizing subsheaves of rank 1 . If $\mathcal{E}$ is such a subsheaf, we can embed it into its reflexive hull $\mathcal{E}^{\vee \vee}$. This is a reflexive rank 1 sheaf, that is, a line bundle. Since $\mathcal{F}^{[2]}$ is locally free, it is also reflexive. Now $\mathcal{E}^{\vee \vee}$ is a subbundle of $\mathcal{F}^{[2]}$, and the first Chern classes of $\mathcal{E}^{\vee \vee}$ and $\mathcal{E}$ coincide. Therefore, $\mathcal{E}^{\vee \vee}$ is destabilizing. This gives a contradiction to Theorem 3.4.

We can generalize this result to arbitrary torsion-free rank 1 sheaves on $X$ with nonvanishing first Chern class, as follows.

THEOREM 4.2. Let $\mathcal{F}$ be a torsion-free rank 1 sheaf on $X$ satisfying $c_{1}(\mathcal{F}) \neq 0$. Then for sufficiently large $N, \mathcal{F}^{[2]}$ is a $\mu_{H_{N}}$-stable rank 2 torsionfree sheaf on $X^{[2]}$.

Proof. Every torsion-free rank 1 sheaf $\mathcal{F}$ on a surface can be written as $\mathcal{F} \simeq \mathcal{L} \otimes \mathcal{I}_{Z}$ for some line bundle $\mathcal{L}$ and an ideal sheaf $\mathcal{I}_{Z}$ of a 0 -dimensional subscheme $Z \subset X$. We thus have an injection $\mathcal{F} \subseteq \mathcal{L}$, and of course, $c_{1}(\mathcal{F})=$ $c_{1}(\mathcal{L})$. In particular, the line bundle $\mathcal{L}$ is not trivial.

Now since $(-)^{[2]}$ is an exact functor, we see (cf. [Sc, Lemma 23]) that $\mathcal{F}^{[2]}$ is also torsion-free. Furthermore, we have an injection $\mathcal{F}^{[2]} \subseteq \mathcal{L}^{[2]}$. But $c_{1}\left(\mathcal{F}^{[2]}\right)=c_{1}\left(\mathcal{L}^{[2]}\right)$ because the cokernel of the inclusion $\mathcal{F}^{[2]} \hookrightarrow \mathcal{L}^{[2]}$ is $\mathcal{O}_{Z}^{[2]}$, which is supported in codimension 2. Thus, the stability of $\mathcal{F}^{[2]}$ follows immediately.

Now we want to consider the case $r=\operatorname{rk} \mathcal{F}=2$. We have seen before that $\mathcal{F}^{[2]}$ cannot contain destabilizing line subbundles. In this section we will prove that in most cases, in fact, $\mathcal{F}^{[2]}$ does not contain any destabilizing subsheaves. We start with a technical lemma.

LEMmA 4.3. Let $(Y, \mathcal{O}(1))$ be a polarized smooth projective variety, and let $\mathcal{H}$ be a pure sheaf on $Y$. The maximal destabilizing subsheaf of $\mathcal{H}$ is saturated. 
Proof. Denote by $\mathcal{H}^{\prime}$ the maximal destabilizing subsheaf. Its saturation $\mathcal{H}_{\text {sat }}^{\prime}$ in $\mathcal{H}$ is a subsheaf of $\mathcal{H}$ of the same rank containing $\mathcal{H}^{\prime}$. Therefore, $\mu\left(\mathcal{H}_{\text {sat }}^{\prime}\right) \geq \mu\left(\mathcal{H}^{\prime}\right)$. By the maximality of $\mathcal{H}^{\prime}$, we must have $\mathcal{H}_{\text {sat }}^{\prime} \simeq \mathcal{H}^{\prime}$.

TheOREM 4.4. Let $\mathcal{F}$ be a rank $2 \mu_{H}$-stable vector bundle on $X$, and assume that $f=c_{1}(\mathcal{F}) \neq 0$. Then for sufficiently large $N, \mathcal{F}^{[2]}$ is a $\mu_{H_{N}}{ }^{-}$ stable rank 4 vector bundle on $X^{[2]}$.

Proof. Let $\mathcal{E}$ be the maximal destabilizing subsheaf of $\pi^{\star} \mathcal{F}^{[2]}$. It is semistable and $S_{2}$-linearized. Similar to the proof of Corollary 4.1, one can show that $\mathcal{E}$ is reflexive, and by Lemma 4.3 , we see that it is saturated. By Theorem $3.4, \mathcal{E}$ cannot have rank 1 . Let us first consider the case $r k \mathcal{E}=3$, and let us have a look at the corresponding short exact sequence on $\widehat{X \times X}$,

$$
0 \rightarrow \mathcal{E} \rightarrow \pi^{\star} \mathcal{F}^{[2]} \rightarrow \mathcal{Q} \rightarrow 0
$$

where $\mathcal{Q}$ is the corresponding destabilizing quotient. Let us write $c_{1}(\mathcal{E})=$ $e \otimes 1+1 \otimes e+a D$. Using (2), we see that the dual of this sequence is the following:

$$
\begin{aligned}
& 0 \rightarrow \underbrace{\mathcal{H o m}_{\mathcal{O}_{\widetilde{X \times X}}}\left(\mathcal{Q}, \mathcal{O}_{\widetilde{X \times X}}\right)}_{=: \mathcal{Q}^{\prime}} \rightarrow \pi^{\star}\left(\mathcal{F}^{\vee[2]}\right) \otimes \mathcal{L}_{(0,0,1)} \\
& \rightarrow \mathcal{E}^{\vee} \rightarrow \mathcal{E} x t_{\mathcal{O}_{\widetilde{X \times X}}^{1}}\left(\mathcal{Q}, \mathcal{O}_{\widetilde{X \times X}}\right) \rightarrow 0 .
\end{aligned}
$$

Since $\mathcal{E}$ is saturated, then $\mathcal{Q}$ is torsion-free, and so the support of $\mathcal{E} x t_{\mathcal{O}_{\widetilde{X} \times X}}^{1}(\mathcal{Q}$, $\mathcal{O}_{\widetilde{X \times X}}$ ) has codimension at least 2 , so it is vanishing first Chern class. We compute

$$
\begin{aligned}
c_{1}\left(\mathcal{Q}^{\prime}\right) & =c_{1}\left(\pi^{\star}\left(\left(\mathcal{F}^{\vee}\right)^{[2]}\right)\right)+c_{1}\left(\mathcal{L}_{(0,0,1)}\right) \cdot \operatorname{rk}\left(\pi^{\star}\left(\left(\mathcal{F}^{\vee}\right)^{[2]}\right)\right)-c_{1}\left(\mathcal{E}^{\vee}\right) \\
& =(e-f) \otimes 1+1 \otimes(e-f)+(a+2) D .
\end{aligned}
$$

Now we may assume that $\mathcal{Q}^{\prime}$ is reflexive, that is, locally free. (If necessary, we replace $\mathcal{Q}^{\prime}$ by its reflexive hull, which still gives a subsheaf of $\pi^{\star}\left(\mathcal{F}^{\vee[2]}\right) \otimes$ $\mathcal{L}_{(0,0,1)}$ with the same first Chern class.) More precisely, $\mathcal{Q}^{\prime} \simeq \mathcal{L}_{(e-f, e-f, a+2)}$. We have an inclusion $\mathcal{Q}^{\prime} \otimes \mathcal{L}_{(0,0,-1)} \hookrightarrow \pi^{\star} \mathcal{F}^{\vee[2]}$. Now, by Lemma 2.5 , the destabilizing condition on $\mathcal{E}$ implies that

$$
4 H . e \geq 3 H . f .
$$


Thus, $2 H .(e-f) \geq-(H . f / 2)$, and since $\mathcal{Q}^{\prime} \otimes \mathcal{L}_{(0,0,-1)} \subset r_{1}^{\star} \mathcal{F}^{\vee}$, then by Corollary 3.2 we get a contradiction.

Finally, assume that the maximal destabilizing subsheaf of $\pi^{\star} \mathcal{F}^{[2]}$ is a rank 2 sheaf $\mathcal{E}$. Again, its first Chern class can be written as $c_{1}(\mathcal{E})=e \otimes$ $1+1 \otimes e+a D$ with $e \in \operatorname{Pic} X$ and $a \in \mathbb{Z}$, and by the fundamental exact sequence (3) we get an injective $S_{2}$-equivariant homomorphism $\mathcal{E} \hookrightarrow r_{1}^{\star} \mathcal{F} \oplus$ $r_{2}^{\star} \mathcal{F}$. We will denote its composition with the projection onto the first factor by $\beta: \mathcal{E} \rightarrow r_{1}^{\star} \mathcal{F}$. Now we distinguish three cases.

(a) $\operatorname{rank} \operatorname{ker} \beta=0$

Thus, $\operatorname{ker} \beta$ is a torsion subsheaf of $\mathcal{E}$, so it is trivial since $\mathcal{E}$ is torsion-free. Thus, $\beta$ is an isomorphism away from an effective divisor $j: Y \hookrightarrow \widehat{X \times X}$. Thus, $\operatorname{coker} \beta$ can be written as $j_{\star} \mathcal{K}$ for some sheaf $\mathcal{K}$ on $Y$. Let $Y=\bigcup_{i} Y_{i}$ be the decomposition into irreducible components; then, by Proposition 2.3, we can write its first Chern class as $c_{1}(\operatorname{coker} \beta)=\sum_{i}\left(Y_{i} \cdot \operatorname{rk} \mathcal{K}_{i}\right)$, where $\mathcal{K}_{i}$ is the restriction of $\mathcal{K}$ to $Y_{i}$. On the other hand, we can compute the first Chern class of coker $\beta$ directly:

$$
c_{1}(\operatorname{coker} \beta)=c_{1}\left(r_{1}^{\star} \mathcal{F}\right)-c_{1}(\mathcal{E})=f \otimes 1-e \otimes 1-1 \otimes e-a D .
$$

Now $Y$ is effective. Thus, if $\operatorname{rk} \mathcal{K}_{i} \neq 0$ for some $i$, we must have $(f-e) \otimes 1-$ $1 \otimes e-a D$ effective. Evaluating against the polarization $\widetilde{H_{N}}$ yields $2 H . e<$ $H . f$. Together with the destabilizing condition on $\mathcal{E}$ - which implies that 2.He $\geq H$. $f$-we get a contradiction. If $\operatorname{rk} \mathcal{K}_{i}=0 \forall i$, that is, if $c_{1}(\operatorname{coker} \beta)=$ 0 , then we must have $f=0$, which we excluded.

(b) $\operatorname{rank} \operatorname{ker} \beta=2$

This says that on an open subset $\beta$ has to vanish, which by symmetry contradicts the fact that $\mathcal{E}$ injects into $r_{1}^{\star} \mathcal{F} \oplus r_{2}^{\star} \mathcal{F}$.

(c) $\operatorname{rank} \operatorname{ker} \beta=1$

Now $\operatorname{im} \beta$ is a rank 1 quotient sheaf of $\mathcal{E}$, and we write its first Chern class $c_{1}(\operatorname{im} \beta)=g \otimes 1+1 \otimes h+b D$. The semistability of $\mathcal{E}$ yields

$$
H . e \leq H .(g+h) .
$$

At the same time, $\operatorname{im} \beta$ is a rank 1 subsheaf of $r_{1}^{\star} \mathcal{F}$. Denote by $\operatorname{im} \beta^{\vee \vee}$ its reflexive hull. This is a reflexive rank 1 sheaf and thus a line bundle. And it has the same first Chern class as $\operatorname{im} \beta$, so im $\beta^{\vee \vee}=\mathcal{L}_{(g, h, b)}$. The destabilizing condition on $\mathcal{E}$ implies that $2 H . e \geq H$.f. Putting things together, we 
find a line subbundle $\mathcal{L}_{(g, h, b)}$ in $r_{1}^{\star} \mathcal{F}$ satisfying $2 H$. $(g+h) \geq H$.f. This is a contradiction to Corollary 3.2.

\section{$\S 5$. The case of the trivial line bundle}

In the previous section we explicitly excluded the case $\mathcal{F} \simeq \mathcal{O}_{X}$. In fact, we have the following result.

Proposition 5.1. The tautological vector bundle $\mathcal{O}_{X}^{[2]}$ associated with the trivial line bundle $\mathcal{O}_{X}$ is not $\mu_{H_{N}}$-stable for sufficiently large $N$.

Proof. By [D, Théorème 1], we have $H^{0}\left(\mathcal{O}_{X}^{[2]}\right) \cong \mathbb{C}$. Thus, the structure sheaf $\mathcal{O}_{X^{[2]}}$ is a line subbundle of $\mathcal{O}_{X}^{[2]}$. We compare the slopes in order to show that $\mathcal{O}_{X^{[2]}}$ is destabilizing. By Lemma 2.5, we have

$$
\begin{aligned}
\mu_{\widetilde{H_{N}}}\left(\pi^{\star} \mathcal{O}_{X^{[2]}}\right) & =0, \\
\mu_{\widetilde{H_{N}}}\left(\pi^{\star} \mathcal{O}_{X}^{[2]}\right) & =-6 H^{2} N^{2}+O(N) .
\end{aligned}
$$

Thus, we see that for sufficiently large $N$, the subbundle $\mathcal{O}_{X^{[2]}}$ is destabilizing.

Acknowledgments. I thank Klaus Hulek, David Ploog, Marc NieperWisskirchen, and Andreas Krug for many useful comments and suggestions. Special thanks go to one of the referees for helping me improve this paper considerably.

\section{REFERENCES}

[D] G. Danila, Sur la cohomologie d'un fibré tautologique sur le schéma de Hilbert d'une surface, J. Algebraic Geom. 10 (2001), 247-280. MR 1811556.

[EGL] G. Ellingsrud, L. Göttsche, and M. Lehn, On the cobordism class of the Hilbert scheme of a surface, J. Algebraic Geom. 10 (2001), 81-100. MR 1795551.

[F] W. Fulton, Intersection Theory, Ergeb. Math. Grenzgeb. (3) 2, Springer, Berlin, 1984. MR 0732620.

[H] R. Hartshorne, Algebraic Geometry, Grad. Texts in Math. 52, Springer, New York, 1977. MR 0463157.

[M] S. Mukai, Symplectic structure of the moduli space of sheaves on an abelian or K3 surface, Invent. Math. 77 (1984), 101-116. MR 0751133.

[Sc] L. Scala, Some remarks on tautological sheaves on Hilbert schemes of points on a surface, Geom. Dedicata 139 (2009), 313-329. MR 2481854. DOI 10.1007/ s10711-008-9338-x.

[Sch] U. Schlickewei, Stability of tautological vector bundles on Hilbert squares of surfaces, Rend. Semin. Mat. Univ. Padova 124 (2010), 127-138. MR 2752679. DOI 10.4171/RSMUP/124-7. 
[W] M. Wandel, Tautological sheaves: Stability, moduli spaces and restrictions to generalised Kummer varieties, preprint, arXiv:1308.4304v1 [math.AG].

Research Institute for Mathematical Sciences

Kyoto University

Kyoto 606-8502

Japan

wandel@math. uni-hannover.de 